\title{
Semiclassical approximation in Batalin-Vilkovisky formalism.
}

\author{
Albert Schwarz \\ Department of Mathematics, University of California, \\ Davis, CA 95616 \\ ASSCHWARZ@UCDAVIS.EDU
}

October 15, 2018

\begin{abstract}
The geometry of supermanifolds provided with a $Q$-structure (i.e. with an odd vector field $Q$ satisfying $\{Q, Q\}=0$ ), a $P$-structure (odd symplectic structure ) and an $S$-structure (volume element) or with various combinations of these structures is studied. The results are applied to the analysis of the Batalin-Vilkovisky approach to the quantization of gauge theories. In particular the semiclassical approximation in this approach is expressed in terms of Reidemeister torsion.
\end{abstract}

\section{Introduction.}

The Batalin-Vilkovisky formalism (BV-formalism) is based on a notion of an odd Poisson bracket (antibracket). The odd Poisson bracket of two functions $F, G$ on the (super)space $R^{n, n}$ with even coordinates $x^{1}, \ldots, x^{n}$ and odd coordinates $\xi_{1}, \ldots, \xi_{n}$ can be defined by the formula

$$
\{F, G\}=\frac{\partial F}{\partial x^{a}} \frac{\partial_{l} G}{\partial \xi_{a}}-\frac{\partial_{r} F}{\partial \xi_{a}} \frac{\partial G}{\partial x^{a}}
$$

The transformations of $R^{n, n}$ preserving the bracket (1) are called odd symplectic transformations or $P$-transformations. Volume preserving $P$-transformations are called $S P$-transformations. A manifold $X$ pasted together from domains in $R^{n, n}$ by means of $P$-transformations is called an odd symplectic manifold or a $P$-manifold. Replacing $P$-transformations by $S P$-transformations in this definition we get a notion of an $S P$-manifold. In a $P$-manifold $X$ we have a notion of an odd Poisson bracket $\{F, G\}$ of two functions on $X$; in an arbitrary coordinate system we can express $\{F, G\}$ as

$$
\{F, G\}=\frac{\partial_{r} F}{\partial z^{i}} \omega^{i j}(z) \frac{\partial_{l} G}{\partial z^{j}} .
$$


The 2-form $\omega=d z^{i} \omega_{i j}(z) d z^{j}$ is closed. (Here the matrix $\omega_{i j}$ is inverse to $\omega^{i j}$.) The formula

$$
\omega(\zeta, \tilde{\zeta})=\zeta^{i} \omega_{i j}(z) \tilde{\zeta}^{j}
$$

determines an odd inner product in the space $T_{z}(X)$ of tangent vectors to $X$ at the point $z \in X$. A Lagrangian submanifold $L$ of $X$ is by definition a $(k, n-k)$ dimensional submanifold of $X$ where the form $\omega$ vanishes (i.e. $\omega(\zeta, \tilde{\zeta})=0$ for all pairs $\zeta, \tilde{\zeta}$ of tangent vectors $\left.\zeta, \tilde{\zeta} \in T_{z}(L)\right)$. In other words $L$ is a $(k, n-k)$ dimensional isotropic submanifold of $X$.

The volume element in an $S P$-manifold $X$ generates a volume element $\nu$ in every tangent space $T_{z}(X)$, i.e. a function $\nu\left(e_{1}, \ldots, e_{2 n}\right)$ satisfying $\nu\left(\tilde{e}_{1}, \ldots, \tilde{e}_{2 n}\right)=$ $\operatorname{det} a \cdot \nu\left(e_{1}, \ldots, e_{2 n}\right)$.

(Here $e_{1}, \ldots, e_{2 n}$ and $\tilde{e}_{1}, \ldots, \tilde{e}_{2 n}$ are bases of $T_{z}(X)$ connected by the formula $\left.\tilde{e}_{i}=a_{i}^{j} e_{j}.\right)$. One can define a volume element $\lambda$ in a Lagrangian manifold $L \subset X$; namely if $e_{1}, \ldots, e_{n}$ is a basis of $T_{z} L$ we take

$$
\lambda\left(e_{1}, \ldots, e_{n}\right)=\mu\left(e_{1}, \ldots, e_{n}, f^{1}, \ldots, f^{n}\right)^{1 / 2}
$$

where $f^{1}, \ldots, f^{n}$ satisfy $\omega\left(e_{i}, f^{j}\right)=\delta_{i}^{j}$. We will assume always that all manifolds under consideration are compact. Let us define an operator $\Delta$ on an $S P$-manifold $X$ by the formula

$$
\Delta H=\operatorname{div} K_{H}
$$

where the divergence is determined by the volume element in $X$ and $K_{H}^{i}=$ $\omega^{i j} \partial_{l} H / \partial z^{j}$. The Batalin-Vilkovisky approach to quantization of gauge theories is based on the consideration of the integrals having the form

$$
\int_{L} H d \lambda
$$

where $\Delta H=0$ and $L$ denotes the Lagrangian submanifold of $X$. It is proved in [1] that (2) does not change by a continuous deformation of $L$; more generally (2) does not change when $L$ is replaced by another Lagrangian manifold $L^{\prime}$ from the same homology class; see [2]. (Saying that two submanifolds $L$ and $L^{\prime}$ of a supermanifold $X$ belong to the same homology class we have in mind that their bodies $m(L)$ and $m\left(L^{\prime}\right)$ are homologous in the body $m(X)$ of $\left.X\right)$. In physical applications the integrand $H$ in (2) has the form $H=\exp \left(-\hbar^{-1} S\right\}$ where $S$ is an extension of the classical action; the choice of the Lagrangian manifold $L$ corresponds to the choice of the gauge condition.

One of our aims is to calculate the asymptotic behavior of $\int_{L} \exp \left(-\hbar^{-1} S\right) d \lambda$ as $\hbar \rightarrow 0$ (semiclassical approximation). It is important to emphasize that the gauge condition (the choice of Lagrangian submanifold) will not enter directly into the answer. One of the possible expressions of the answer involves a generalization of the so called Reidemeister torsion. The present paper is devoted 
to the case of finite-dimensional integrals; however one can develop a similar technique for infinite- dimensional integrals. This technique is based on the results about the infinite- dimensional version of Reidemeister torsion (Ray-Singer torsion) proved in [4]. In particular it is useful for the calculation of anomalies in the BV-approach.

Our proofs are based on some general results about $S P$-manifolds and about Reidemeister torsion. These results are interesting independently of applications to the study of semiclassical approximation. One can consider the corresponding part of the present paper as the next step in the analysis of the geometry of BV-quantization after the paper [2].

The paper is organized as follows. In Section 1 we state the formula for semiclassical approximation in BV-formalism and give a proof of this formula. The proof is based on the theorems proved later, therefore the reader interested not only in the main ideas, but also in complete understanding of the proof is recommended to read at first Sections 2-7 and then come back to Section 1. Sections 3-6 are devoted to the theory of (super)manifolds provided with $Q$-structure (i.e. with an odd vector field $Q$ satisfying $\{Q, Q\}=0), P$-structure (odd symplectic structure) and $S$-structure (volume element) or with various combinations of these structures. The structures studied in these sections can be considered as a geometric basis of the BV-formalism. For example, $Q P$ structure is connected with master equation and $Q S P$-structure is related to the quantum version of this equation. In Section 2 we study a generalization of Reidemeister torsion. In Section 7 we analyze the torsion of operators acting on linear $P$-manifolds.

In the present paper we use the definitions and results of the paper [2], however one can read this paper independently of [2].

Notations. The symbol $R^{p, q}$ denotes $(p, q)$-dimensional linear superspace. The body of a supermanifold $X$ will be denoted by $m(X)$. The tangent space to $X$ at the point $x \in X$ is denoted by $T_{x} X$. We almost always omit the prefix "super". In particular we write "manifold" instead of "supermanifold", "determinant" instead of "superdeterminant" (Berezinian). The notations $H_{k}(X), H^{k}(X)$ are used for homology and cohomology groups of $X$ with coefficients in the field of real numbers. All functions, mappings etc. are supposed to be smooth.

\section{Semiclassical approximation.}

Let us consider an $S P$-manifold $X$, a function $S$ on $X$ and a Lagrangian submanifold $L \subset X$.

Let us suppose that $H=\exp \left(-\hbar^{-1} S\right)$ satisfies the equation $\Delta H=0$ for every $\hbar$; then $S$ obeys the equation $\{S, S\}=0$ (master equation) and the condition $\Delta S=0$. Let us denote by $R$ the set of critical points of the restriction $\tilde{S}$ of $S$ to the Lagrangian manifold $L$. One can check that the vector field $K_{S}^{i}=$ $\omega^{i j} \partial_{l} S / \partial z^{j}$ corresponding to $S$ is an odd vector field satisfying $\left\{K_{S}, K_{S}\right\}=0$ and tangent to $R$ (i.e. in the terminology of Section $3, K_{S}$ determines $Q$ - 
structure both in $X$ and $R$.) The set of zeros of the vector field $K_{S}$ coincides with the set $Y$ of all critical points of $S$. We would like to study the asymptotic behavior of the integral of $H$ over $L$ as $\hbar \rightarrow 0$. It is well known that this behavior can be expressed in terms of an integral over $R$ (see Lemma 2). The integrand of this integral is $K_{S}$-invariant, hence the integral over $R$ can be reduced to the integral over a neighborhood of $R \cap Y$ (see[5] or Lemma 5). Therefore in principle we can express the asymptotic behavior of $\int_{L} \exp \left(-\hbar^{-1} S\right) d \lambda$ as $\hbar \rightarrow 0$ in terms of the behavior of the function $S$ in the neighborhood of the set $Y$ of all critical points of $S$. Let us give the corresponding expression under some conditions on the dimension of $L$. For every point $x \in Y$ we define an operator $Q_{x}$ acting on the tangent space $T_{x} Y$ as an operator with the matrix $\omega^{i j} S_{j k}$ where $S_{j k}$ denotes the Hessian of $S$ at the point $x$. It is easy to check that $Q_{x}^{2}=0$ and therefore we can consider the homology group $H_{x}=Z_{x} / B_{x}$ where $Z_{x}=\operatorname{Ker} Q_{x}, \quad B_{x}=\operatorname{Im} Q_{x}$. It is easy to check that $Z_{x} \subset T_{x} Y$; we will suppose that $Y$ is a manifold and $Z_{x}=T_{x} Y$. Therefore there exists a natural map of $T_{x} Y$ onto $H_{x}$; we will denote this map by $\pi_{x}$. The dimensions of $X$ and $Y$ will be denoted by $(n, n)$ and $\left(z_{1}, z_{2}\right)$ correspondingly, the dimension of $B_{x}$ will be denoted by $\left(b_{1}, b_{2}\right)$. It is important to emphasize that in the situations arising in physics the set $Y$ is not necessarily a manifold.

Let us introduce the notion of a regular critical submanifold of $X$ as follows. We will say that $C$ is a regular critical submanifold if $C$ is a compact submanifold of $Y$ and for every point $x \in C$ one can find vectors $e_{1}, \ldots, e_{q}, f^{1}, \ldots, f^{q} \in T_{x} Y$ in such a way that $e_{1}, \ldots, e_{q} \in T_{x} C, \omega\left(e_{i}, e_{j}\right)=0, \omega\left(e_{i}, f^{j}\right)=\delta_{i}^{j}$ and the vectors $\pi_{x}\left(e_{1}\right), \ldots, \pi_{x}\left(e_{q}\right), \pi_{x}\left(f^{1}\right), \ldots, \pi_{x}\left(f^{q}\right)$ constitute a basis in $H_{x}$. It follows from this definition that for every $x \in C$ one can find vectors $g_{1}, \ldots, g_{n-k} \in T_{x} X$ in such a way that the vectors $\left(e_{1}, \ldots, e_{q}, f^{1}, \ldots, f^{q}, g_{1}, \ldots, g_{n-q}, Q_{x} g_{1}, \ldots, Q_{x} g_{n-q}\right)$ form together a basis in $T_{x} X$. This remark permits us to define a volume element in $T_{x} C$ (and therefore in $C$ ) by the formula

$$
\gamma\left(e_{1}, \ldots, e_{q}\right)=\mu\left(e_{1}, \ldots, e_{q}, f^{1}, \ldots, f^{q}, g_{1}, \ldots, g_{n-q}, Q_{x} g_{1}, \ldots, Q_{x} g_{n-q}\right)^{1 / 2}
$$

Therefore we can talk about the volume of a regular critical manifold $C$.

Theorem 1. The asymptotic behavior as $\hbar \rightarrow 0$ of the integral of $\exp \left(-\hbar^{-1} S\right)$ over an $(l, n-l)$-dimensional Lagrangian manifold $L$ can be described as follows:

$$
\int_{L} \exp \left(-\hbar^{-1} S\right) d \lambda \approx \hbar^{\left(z_{1}-z_{2}\right) / 2} \int_{C} \exp \left(-\hbar^{-1} S\right) d \gamma
$$

where $C$ is an appropriate regular critical submanifold of $X$. More precisely, the homology class $[C]$ of $m(C)$ must be connected with the homology class $[L]$ of $m(L)$ by the formula

$$
D[C]=i^{*} D[L]
$$

Here $D$ denotes the Poincare duality between homology and cohomology groups of a manifold and $i^{*}$ is a homomorphism of cohomology groups induced 
by the embedding $i: Y \rightarrow X$. In such a way $[L] \in H_{l}(m(X)), \quad D[L] \in$ $H^{n-l}(m(X)), \quad[C] \in H_{z_{1}+l-n}(m(Y)), \quad D[C] \in H^{n-l}(m(Y))$. We suppose that $z_{2} \geq l \geq b_{2}, n-l \geq b_{1}$.

It is obvious that $S$ is constant on every connected component of $Y$. Therefore in the case when $C$ is connected or belongs to a connected component of $Y$ the right hand side of (4) is equal to

$$
\hbar^{\left(z_{1}-z_{2}\right) / 2} \exp \left(-\hbar^{-1} S_{0}\right) \cdot \operatorname{volume}(C)
$$

where $S_{0}$ denotes the value of $S$ on $C$. In the general case we get a sum over the components of $C$. (Note that we did not assume that $S$ is real.)

Theorem 1 can be formulated in a more invariant form in the following way. First of all we note that the restriction $\tilde{\omega}$ to $Y$ of the 2-form $\omega$ corresponding to the $P$-structure on $X$ is a degenerate odd closed 2-form on $Y$. Factorizing $Y$ with respect to null-vectors of $\tilde{\omega}$ we obtain a manifold $Y^{\prime}$ provided with a nondegenerate odd closed 2 -form and therefore with a $P$-structure. (The manifold $Y^{\prime}$ does not always exist; see Section 5 for details.) One can identify the tangent spaces to $Y^{\prime}$ with the homology groups $H_{x}=\operatorname{Ker} Q_{x} / \operatorname{Im} Q_{x}, \quad x \in Y$. This permits us to introduce a volume element in $Y^{\prime}$ using the construction of torsion. (This construction allows us to define a volume element in $H_{x}$ starting with the volume element in $T_{x} X$; see Section 2). One can prove that this volume element together with the $P$-structure in $Y^{\prime}$ determines an $S P$-structure on $Y^{\prime}$; see Section 6. The image $C^{\prime}$ of a regular critical submanifold $C$ of $X$ under the natural projection $Y \rightarrow Y^{\prime}$ is a Lagrangian submanifold of the $S P$-manifold $Y^{\prime}$ (the manifold $C^{\prime}$ can have self-intersections). Therefore we can calculate the volume of $C^{\prime}$ as a volume of a Lagrangian submanifold of an $S P$-manifold. This permits us to give an alternative formulation of Theorem 1; namely in the case when $S$ is constant on $C$ we can use (6) replacing the volume $(C)$ by the volume $\left(C^{\prime}\right)$. (Combining the definition of torsion and the definition of a volume element in a Lagrangian submanifold we obtain that volume $(C)=\operatorname{volume}\left(C^{\prime}\right)$ ).

The reformulation of the Theorem 1 permits us to use the results of [2] to prove this theorem. Namely it follows immediately from Theorem 1 of [2] that the left hand side of (4) depends only on the homology class of Lagrangian manifold L and the right hand side of (4) depends only on the homology class of the regular critical submanifold $C$.

Now we can apply the following lemma.

Lemma 1.

Let us consider an $(n, n)$-dimensional $P$-manifold $X$ and a function $S$ on $X$, satisfying $\{S, S\}=0$. Let us suppose that a Lagrangian submanifold $L$ of $X$ is in general position with respect to the manifold $Y$ of critical points of $S$ (i.e. $L \cap Y$ is a manifold and for $x \in L \cap Y$ we have $T_{x}(L \cap Y)=T_{x} L \cap T_{x} Y, \quad T_{x} L+T_{x} Y=$ $\left.T_{x} X\right)$. Then $L \cap Y$ is a regular critical submanifold of $X$.

To prove this lemma we note that a non-zero vector $b \in B_{x}, \quad x \in L \cap Y$ cannot belong to $T_{x}(L \cap Y)$. Really, $b \in B_{x}$ is orthogonal to $Z_{x}=T_{x} Y$. If 
$b \in T_{x} L$, it is orthogonal to $T_{x} L$ because $T_{x} L$ is isotropic. We see that a vector $b \in B_{x} \cap T_{x} L$ is orthogonal to $T_{x} X=T_{x} L+T_{x} Y$; hence $b=0$ (because the inner product in $T_{x} X$ is non-degenerate). The manifold $L \cap Y$ is isotropic; it follows from the consideration above that its image $\pi(L \cap Y)$ under the natural projection $\pi: Y \rightarrow Y^{\prime}$ is an isotropic manifold of the same dimension (more precisely $\pi$ is an immersion of $L \cap Y$ into $Y^{\prime}$ ). Calculating the dimension of $L \cap Y$ we conclude that $\pi(L \cap Y)$ is a Lagrangian submanifold of $Y^{\prime}$ and therefore $L \cap Y$ is a regular critical submanifold. (If $\operatorname{dim} X=(n, n), \operatorname{dim} Y=\operatorname{dim} Z_{x}=\left(z_{1}, z_{2}\right)$, $\operatorname{dim} B_{x}=\left(b_{1}, b_{2}\right), \operatorname{dim} L=(l, n-l)$ we have $\operatorname{dim} L \cap Y=\left(z_{1}+l-n, z_{2}-l\right)$, $\operatorname{dim} Y^{\prime}=\left(z_{1}-b_{1}, z_{2}-b_{2}\right)=\left(z_{1}+z_{2}-n, z_{1}+z_{2}-n\right)$. Taking into account that $\left(z_{1}+l-n\right)+\left(z_{2}-l\right)=z_{1}+z_{2}-n$ we see that $\pi(L \cap Y)$ is a Lagrangian submanifold of $Y^{\prime}$ ). In the proof above we considered for simplicity the case when the $P$-manifold $Y^{\prime}$ exists. However this restriction is unnecessary. The statement of the lemma is local, hence we always can work in a neighborhood of $x \in L \cap Y$ where our reasoning is valid.

To prove Theorem 1 we consider at first the case when the Lagrangian manifold $L$ is in general position with respect to $Y$ and all critical points of the restriction of $S$ to $L$ are critical points of $S$ on $X$ (i. e. belong to $Y$ ). Applying Lemma 2 we express the asymptotics of (4) as $\hbar \rightarrow 0$ in terms of an integral over the set $R$ of critical points of the restriction of $S$ to $L$; in the case at hand $R$ coincides with the regular critical submanifold $C=L \cap Y$. The integrand can be represented as a partition function of a quadratic functional $S_{x}$ (of the Hessian of $S$ at the point $x \in C$ ); see Lemma 2. This partition function can be expressed in terms of torsion; see Lemma $7^{\prime \prime}$. Using this fact we obtain the statement of Theorem 1 (or more precisely, the equivalent statement formulated above) in the case at hand. Essentially the same arguments combined with Lemma 5 can be applied if we replace the condition $R=L \cap Y$ with the weaker condition that for every $x \in C=L \cap Y$ the subspace $N_{x}$ of zero modes of the restriction of $S_{x}$ to $T_{x} L$ coincides with $T_{x} C=T_{x} L \cap T_{x} Y$. Moreover, if this condition is violated on a subset of $C$ having zero measure we still can arrive at the same conclusion.

If $L$ is an arbitrary Lagrangian submanifold of $X$ and $z_{2} \geq l$ then in every neighborhood of $L$ we can find a Lagrangian submanifold $\tilde{L}$ in general position with respect to $Y$. It follows from Lemma 9 that in the case when $l \geq b_{2}, n-l \geq$ $b_{1}$ we can choose $\tilde{L}$ in such a way that the arguments above give a proof of (4) with $L$ replaced by $\tilde{L}$. This proves (4) for arbitrary $L$, because neither side of (4) changes when we replace $L$ by $\tilde{L}$.

Let us formulate in conclusion a topological theorem leading to equation (5). Let us consider an $m$-dimensional manifold $M$ and an $n$-dimensional submanifold $N$. Let us suppose that a $k$-dimensional submanifold $L \subset M$ is in general position with respect to $N$. Then the intersection $L \cap N$ is a manifold. We will suppose that $M, N$ and $L$ are oriented compact manifolds. Then one can consider homology classes $[L] \in H_{k}(M),[L \cap N] \in H_{k+n-m}(N)$ of $L$ and $L \cap N$ and their Poincare duals $D[L \cap N] \in H^{m-k}(N)$ and $D[L] \in H^{m-k}(M)$. It is 
well known that by an appropriate choice of orientation of $L \cap N$ we have

$$
D[L]=j^{*} D[L \cap N]
$$

where $j^{*}$ denotes the homomorphism of cohomology groups corresponding to the embedding $j: N \rightarrow M$ (see for instance [6]). To get (5) we have to apply this formula to the embedding of the body of $Y$ into the body of $X$ induced by the embedding $i: Y \rightarrow X$. It is important to emphasize that the homology classes of regular critical submanifolds arising in the right hand side of (4) can be characterized in purely topological terms and the $S P$-structure on $X$ does not enter the answer.

\section{Torsion. Definition and main properties.}

Let $E$ denote a (finite-dimensional) linear superspace over $R$. A linear measure $\lambda$ on $E$ is by definition an even function $\lambda(e)$ of the basis $e$ in $E$ satisfying the condition $\lambda(A e)=\operatorname{det} A \cdot \lambda(e)$. (Here Ae denotes the basis in $E$ obtained from $e$ by means of the linear transformation $A$. In other words the bases $e=\left(e_{1}, \ldots, e_{k}\right)$ and $\tilde{e}=A e=\left(\tilde{e}_{1}, \ldots, \tilde{e}_{k}\right)$ are connected by the formula $\tilde{e}_{i}=A_{i}^{k} e_{k}$.) The one-dimensional space of linear measures on $E$ will be denoted by $\Lambda(E)$ or simply by $\Lambda E$. We say that the measure $\lambda$ specifies a volume element in $E$ if $\lambda(e)$ is invertible. (Recall that every element $\lambda$ of a Grassmann algebra can be represented as a sum of its number part $m(\lambda)$ and its nilpotent part $n(\lambda)$. An element $\lambda$ is invertible if $m(\lambda) \neq 0)$. Note that every basis $f$ in $E$ determines a volume element $\lambda_{f}$ in $E$ by means of the condition $\lambda_{f}(f)=1$. It is easy to check that there exist canonical isomorphisms

$$
\begin{aligned}
& \Lambda\left(E^{*}\right)=\Lambda(\Pi E)=\Lambda(E)^{*}, \\
& \Lambda(E)=\Lambda\left(E^{\prime}\right) \otimes \Lambda\left(E / E^{\prime}\right) .
\end{aligned}
$$

Here $E^{*}$ denotes the space dual to $E, \Pi E$ is obtained from $E$ by means of parity reversion and $E^{\prime}$ is a linear subspace of $E$. Let us denote by $Q$ a parity reversing linear operator in $E$ satisfying $Q^{2}=0$. As usual we can define the subspace of cycles $Z$ as the kernel of $Q$ and the subspace of boundaries $B$ as the image of $Q$. The homology group $H=H(E, Q)$ can be defined as the linear superspace $Z / B$. (One has to impose some conditions on $Q$ to guarantee that $Z, B$ and $H$ can be considered as superspaces; we always assume that these conditions are satisfied.) It is easy to check that there exists a canonical isomorphism

$$
\Lambda(E)=\Lambda(H) .
$$

This isomorphism will be denoted by Tor, because it can be considered as a generalization of Reidemeister torsion. To construct this isomorphism we note that it follows from (7), (8) and the relations

$$
H=Z / B, \quad \Pi B=E / Z
$$


that

$$
\Lambda(Z)=\Lambda(H) \otimes \Lambda(B), \Lambda(E)=\Lambda(Z) \otimes \Lambda(\Pi B)=\Lambda(Z) \otimes \Lambda(B)^{*} .
$$

The isomorphism (9) is an immediate consequence of (11).

Usually the torsion is considered in the case when $H=0$ and $E$ is provided with a linear measure $\lambda$ from the very beginning. Then $\Lambda(H)$ can be identified with $R^{1,0}$ and the torsion can be interpreted as an even Grassmann number (the image of $\lambda$ by the isomorphism (9)).

One can give a more explicit description of torsion. Let us fix vectors $e_{1}, \ldots, e_{k} \in E$ in such a way that $b_{1}=Q e_{1}, \ldots, b_{k}=Q e_{k}$ constitute a basis of $B$. If $H=0$ the vectors $e_{1}, \ldots, e_{k}, b_{1}, \ldots, b_{k}$ form a basis of $E$ and the torsion can be defined as

$$
\lambda\left(e_{1}, \ldots, e_{k}, b_{1}, \ldots, b_{k}\right)
$$

where $\lambda$ is a measure in $E$. In general case we add elements $h_{1}, \ldots, h_{s} \in Z$ to $e_{1}, \ldots, e_{k}, b_{1}, \ldots, b_{k}$ to get a basis of $E$. The images $\tilde{h}_{1}, \ldots \tilde{h}_{s}$ of $h_{1}, \ldots, h_{s}$ by the natural map of $Z$ onto $H$ constitute a basis of $H$. We can define a measure $\tilde{\lambda}$ in $H$ corresponding to $\lambda$ by the formula

$$
\tilde{\lambda}\left(\tilde{h}_{1}, \ldots, \tilde{h}_{s}\right)=\lambda\left(e_{1}, \ldots, e_{k}, b_{1}, \ldots b_{k}, h_{1}, \ldots h_{s}\right) .
$$

For every polyhedron $X$ we can consider the space $E$ of all cell chains (or cochains) over $R$ in $X$ (the parity is determined by the dimension of a chain). The boundary (or coboundary) operator changes the dimension by one, therefore it is parity reversing and can play the role of the operator $Q$. The standard basis consisting of cells determines an element $\lambda$ of $\Lambda(E)$. Therefore the isomorphism (9) determines an element of $\Lambda(H)$ (a number in the acyclic case). This construction gives the Reidemeister torsion of $X$. (One can consider also Reidemeister torsion in a little bit more general situation when $E$ consists of chains with local coefficients. The local coefficient system is determined by a representation of $\pi_{1}(X)$.)

It is easy to check some simple properties of torsion. Let us consider a linear subspace $E^{\prime} \subset E$ invariant with respect to an operator $Q$ acting on $E$. Then the operator $Q$ generates an operator $\bar{Q}$ acting on the coset space $F=E / E^{\prime}$. If $Q^{2}=0$ we can define $H\left(E^{\prime}, Q\right), H(E, Q)$, and $H(F, \bar{Q})$. These homology groups are connected by an exact triangle. It follows from this fact that

$$
\Lambda H(E, Q)=\Lambda H\left(E^{\prime}, Q\right) \otimes \Lambda H(F, \bar{Q})
$$

and that

$$
H(E, Q)=H(F, \bar{Q})
$$

in the case when $H\left(E^{\prime}, Q\right)=0$. If $Q$ is parity reversing and the volume elements in $E, E^{\prime}$ and $F$ are fixed we can define torsions of $E, E^{\prime}$ and $F$ as elements of $\Lambda H(E, Q), \Lambda H\left(E^{\prime}, Q\right)$ and $\Lambda H(F, \bar{Q})$ correspondingly. We will assume that the 
volume elements in $E, E^{\prime}$ and $F$ are compatible, i.e. the volume element in $E^{\prime}$ and $F$ generate the volume element in $E$ by means of (8). It is easy to prove that in this case

$$
\operatorname{Tor}(E, Q)= \pm \operatorname{Tor}\left(E^{\prime}, Q\right) \otimes \operatorname{Tor}(F, \bar{Q})
$$

(In the case when $H\left(E^{\prime}, Q\right)=0$ and therefore the groups $H(E, Q)$ and $H(E, \bar{Q})$ coincide one can replace $\otimes$ by common multiplication. In the general case we use the identification (14).)

The notion of torsion is closely related to the notion of a partition function of a degenerate quadratic functional. Let us suppose that $S$ is a quadratic linear functional on a linear superspace $E$ provided with a volume element $\lambda$. In the case when the quadratic form $S$ is non-degenerate the partition function $Z_{S}$ can be defined as the integral

$$
Z_{S}=\int_{E} e^{-S} d \lambda=(2 \pi)^{d(E) / 2}(\operatorname{det} \hat{S})^{-1 / 2}
$$

where $d(E)$ denotes the difference between even and odd dimensions of $E$ and $\hat{S}$ stands for the matrix of quadratic form $S$ in the basis $e_{1}, \ldots, e_{n}$ satisfying $\lambda\left(e_{1}, \ldots, e_{n}\right)=1$. If $S$ is degenerate we define $Z_{S}$ as a volume element on the space $N$ of zero modes of $\hat{S}$. Namely, if $f_{1}, \ldots, f_{k}$ is a basis of $N$ we define

$$
Z_{S}\left(f_{1}, \ldots, f_{k}\right)=Z_{E^{\prime}}
$$

Here $Z_{E^{\prime}}$ stands for the partition function of $S$ restricted to the subspace $E^{\prime} \subset E$ satisfying $E^{\prime}+N=E$. The measure in $E^{\prime}$ is given by the formula $\lambda^{\prime}\left(e_{1}, \ldots, e_{n-k}\right)=\lambda\left(e_{1}, \ldots, e_{n-k}, f_{1}, \ldots, f_{k}\right)$. An explicit formula for $Z_{S}$ can be written as follows:

$$
Z_{S}\left(f_{1}, \ldots, f_{k}\right)=(2 \pi)^{d(E)-d(N)}(\operatorname{det} \sigma)^{-1 / 2}
$$

where $\sigma$ denotes the matrix $\sigma_{i j}=S\left(e_{i}, e_{j}\right) \quad 1 \leq i, j \leq n-k$, and the vectors $e_{1}, \ldots, e_{n-k}$ are chosen in such a way that $\lambda\left(e_{1}, \ldots, e_{n-k}, f_{1}, \ldots, f_{k}\right)=1$. (Here $S(x, y)$ stands for the bilinear form corresponding to the quadratic form $S(x)$.)

Note that the notion of a partition function of a quadratic functional can be used to describe the asymptotic behavior of an integral $\int_{X} \exp \left(-\hbar^{-1} \mathcal{S}\right) d \nu$ as $\hbar \rightarrow 0$. Let us suppose that a function $\mathcal{S}$ is defined on a manifold $X$ provided with a volume element (on an $S$-manifold). The set of all critical points of $\mathcal{S}$ will be denoted by $R$; for the sake of simplicity we assume that $R$ is a compact connected manifold. The Hessian of $\mathcal{S}$ at the point $x \in R$ can be considered as a quadratic form $\mathcal{S}_{x}$ defined on the tangent space $T_{x} X$. It is clear that the elements of $T_{x} R$ are zero modes of $\mathcal{S}_{x}$; we suppose that the space $N_{x}$ of zero modes of $\mathcal{S}_{x}$ coincides with $T_{x} R$ (i.e. $R$ is a non-degenerate critical manifold). Then the partition function $Z_{\mathcal{S}_{x}}$ of a degenerate quadratic functional $\mathcal{S}_{x}$ can be 
considered as a volume element on $N_{x}=T_{x} R$. In such a way we constructed a volume element in $R$; we can calculate therefore the volume of $R$.

Lemma 2. The asymptotic behavior as $\hbar \rightarrow 0$ of the integral of $\exp \left(-\hbar^{-1} \mathcal{S}\right)$ over $X$ can be described by the formula

$$
\int_{X} \exp \left(-\hbar^{-1} \mathcal{S}\right) d \nu \approx \hbar^{(d(Y)-d(X)) / 2} \cdot \exp \left(-\hbar^{-1} \mathcal{S}_{0}\right) \operatorname{volume}(R)
$$

Here $\mathcal{S}_{0}$ denotes the value of $\mathcal{S}$ on $R$. (We assumed that $R$ is connected; therefore $\mathcal{S}$ is constant on $R$.)

Let us suppose now that the quadratic form $S$ is invariant with respect to the parity reversing operator $Q$ (this means that $S(z+Q w)=S(z)$ or, equivalently, $\hat{S} Q=0)$. The space $N$ of zero modes of $S$ is invariant with respect to $Q$; we will assume that $Q^{2}=0$ on $N$. We can consider the homology group $H=H(N, Q)$ of $N$ with respect to $Q$. We will define the partition function $Z_{S, Q}$ as a torsion of $Q$ in $N$ with respect to the measure $Z_{S}$ in $N$. The partition function $Z_{S, Q}$ is a number if $H=0$ and a measure on $H$ (an element of $\Lambda(H)$ ) in the general case. Note that the definition of $Z_{S, Q}$ given above can be applied also to the case when the parity reversing operator $Q$ acts only on $N$ and satisfies $Q^{2}=0$ there. The definition above is prompted by the generalization of the FadeevPopov trick introduced in [4]. Let us suppose that the quadratic form $S$ on $E_{0}$ is invariant with respect to a linear operator $T_{0}$ acting from $E_{1}$ into $E_{0}$ (i.e. $S\left(x+T_{0} y\right)=S(x)$ for every $y \in E_{1}$, or equivalently $\left.\operatorname{Im} T_{0} \subset N=\operatorname{Ker} \hat{S}\right)$. We will assume that $N=\operatorname{Im} T_{0}$ (i.e. the degeneracy of $S$ is due entirely to the symmetry $T_{0}$ ) and that there exists a resolution of $T_{0}$, i.e. a sequence of spaces $E_{0}, E_{1}, \ldots, E_{n}$ provided with volume elements and linear maps $T_{i}: E_{i+1} \rightarrow E_{i}$ obeying the condition $\operatorname{Ker} T_{i-1}=\operatorname{Im} T_{i}$. Let us construct a superspace $E$ taking the direct sum $\sum E_{2 k}$ as the even part of $E$ and the direct sum $\sum E_{2 k+1}$ as the odd part of $E$. Then the operators $T_{i}$ determine a parity reversing operator $Q$ on $E$ and the form $S$ determines a form on $E$ that will be denoted by the same letter (by definition $Q=0$ on $E_{0}$ and $S=0$ on $E_{i}, i>o$ ). It follows from our assumptions that $Q^{2}=0$ and that the homology group of $Q$ on the space of zero modes of $S$ is trivial; therefore we can consider the number $Z_{S, Q}$. It is easy to check that $Z_{S, Q}$ coincides with the partition function of $S$ with respect to the resolution $\left(E_{i}, T_{i}\right)$ as it is defined in [4]. (To be more precise the definition of [4] is given for the infinite-dimensional elliptic case, but one can give an analogous definition in the finite-dimensional case at hand.)

\section{Q-manifolds.}

Let us consider a supermanifold $X$ provided with an odd vector field $Q$ satisfying $\{Q, Q\}=0$ (in other words, the corresponding first order differential operator $\hat{Q}$ obeys $\hat{Q}^{2}=0$ ). We will say that such a vector field specifies a $Q$-structure on $X$ or that $X$ is a $Q$-manifold. Let us denote the set of all points $x \in X$ satisfying $Q x=0$ by $Y$. The vector field $Q$ induces a linear map $Q_{x}$ of 
the tangent space $T_{x} X$ to $X$ at the point $x \in Y$ into itself. The matrix of $Q_{x}$ in local coordinates $\left(z^{1}, \ldots, z^{n}\right)$ is $\partial Q^{a} / \partial z^{b}$. The map $Q_{x}$ is parity reversing and obeys $Q_{x}^{2}=0$, therefore we can consider the homology group $H_{x}=H\left(T_{x}, Q_{x}\right)$ and the torsion of $Q_{x}$. We will suppose that for every $x \in Y$ the kernel $Z_{x}$ and the image $B_{x}$ of the operator $Q_{x}$ are linear superspaces of the same dimension and that $Y$ is a supermanifold. Then the tangent space $T_{x} Y$ to $Y$ at the point $x \in Y$ can be identified with $Z_{x}$. The simplest example of a vector field $Q$ satisfying the conditions above is an odd field with constant coefficients in $R^{m, n}$ (for example a field with $\hat{Q}=\frac{\partial}{\partial \xi^{a}}$ where $\xi^{a}$ denotes one of the odd coordinates in $\left.R^{m, n}\right)$. In this case $Y$ is empty. It follows from the superanalog of Frobenius' theorem that conversely in the case when $Y$ is empty one can find a coordinate system in the $Q$-manifold $X$ such that $\hat{Q}=\partial / \partial \xi^{a}$. A more interesting example is given by the formula

$$
\hat{Q}=\eta^{a} \frac{\partial}{\partial \xi^{a}}
$$

where the coordinates $\xi^{a}$ and $\eta^{a}$ have opposite parity. It is clear that the formula (19) can be used to determine a $Q$-structure on the supermanifold $T N$ of tangent vectors to a supermanifold $N$ (locally a point of $T N$ is described by coordinates $\xi^{a}$ in $N$ and coordinates $\eta^{a}$ of a tangent vector; $\xi^{a}$ and $\eta^{a}$ have opposite parity). This $Q$-structure will be called the standard $Q$-structure on $T N$. Differential and pseudodifferential forms on $N$ can be considered as functions on $T N$. (By definition a differential form is a polynomial function with respect to $\eta^{a}$ and a pseudodifferential form must vanish for $\eta^{a}$ tending to infinity if $\eta^{a}$ is even. If all $\xi^{a}$ are even, all $\eta^{a}$ are odd, these two definitions coincide.) The operator (19) coincides with the differential of a differential (or pseudodifferential) form. The set $Y$ can be identified with $N \subset T N$ in the case at hand. It is easy to check that $H\left(T_{x}, Q_{x}\right)=0$ at every point $x \in N$.

One can check that the form (19) of $\hat{Q}$ is general in some sense. In particular, one can prove the following

Theorem 2. If $X$ is a $Q$-manifold, $Y=\{x \in X \mid Q x=0\}$, then for every $x \in Y$ one can find coordinates $\left(\xi^{a}, \eta^{a}, \zeta^{\alpha}\right)$ in a neighborhood $U$ of $x$ in such a way that $Y \cap U$ is singled out by the equations $\eta^{a}=0$ and $\hat{Q}$ has the form (19) in $Y \cap U$.

Let us say that a vector field $b$ in $Y$ belongs to the set $\mathcal{B}$ if for every $x \in Y$ we have $b(x) \in B_{x}=\operatorname{Im} Q_{x}$. One can check that for the fields $b \in \mathcal{B}, b^{\prime} \in \mathcal{B}$ their (graded) commutator $\left[b, b^{\prime}\right\} \in \mathcal{B}$. The proof is based on the fact that the restriction to $Y$ of the field $[Q, A\}$ belongs to $\mathcal{B}$ for every vector field $A$ in $X$ and that locally every field $b \in \mathcal{B}$ can be represented as a restriction of a field $[Q, A\}$. This follows immediately from the remark that in local coordinates $z^{a}$ the field $[Q, A\}$ has the form $\left(\partial Q^{a} / \partial z^{b}\right) \cdot A^{b}$ at the point $x \in Y$. Using the representations $b=[Q, A\}, b^{\prime}=\left[Q, A^{\prime}\right\}$ and the Jacoby identity we obtain

$$
\left[b, b^{\prime}\right\}= \pm\left[Q,\left[A, b^{\prime}\right\}\right\}
$$


and therefore $\left[b, b^{\prime}\right\} \in \mathcal{B}$. This assertion permits us to use the generalization of Frobenius' theorem to the case of a supermanifold. Utilizing this theorem we get a local coordinate system $\left(u^{1}, \ldots, u^{m}\right)$ in $Y$ in so that in the neighborhood of a point $x \in Y$ the field $b=\left(b^{1}, \ldots, b^{m}\right) \in \mathcal{B}$ if and only if $b^{k+1}=\ldots=b^{m}=0$. (In other words the subspaces $B_{x} \subset T_{x} Y$ determine a foliation of $Y$.) We can extend this coordinate system to a local coordinate system $\left(u^{1}, \ldots, u^{m}, v^{1}, \ldots, v^{s}\right)$ in $X$ in such a way that $Y$ is singled out by equations $v^{1}=\ldots=v^{s}=0$. It is easy to check that the operator $\hat{Q}$ in this coordinate system has the form

$$
\hat{Q}=\sum_{a=1}^{k} \sum_{b=1}^{s} Q_{b}^{a}\left(u^{1}, \ldots, u^{m}\right) v^{b} \partial \varnothing v e r \partial u^{a}+\ldots
$$

where we omitted higher order terms with respect to $v^{1}, \ldots v^{s}$. One can verify that the matrix $Q_{b}^{a}$ must be non-degenerate (and therefore $s=k$ ). This follows from the remark that the kernel of the matrix $Q_{y}$ at the point $y \in Y$ coincides with $Z_{y}=T Y(y)$. Therefore we can introduce new coordinates

$$
\xi^{a}=u^{a}, 1 \leq a \leq k, \zeta^{\alpha}=u^{\alpha+k}, 1 \leq \alpha \leq m-k, \eta^{a}=Q_{b}^{a}(u) v^{b}, 1 \leq a \leq k .
$$

We have

$$
\hat{Q}=\eta^{a} \frac{\partial}{\partial \xi^{a}}+(\text { higher order terms with respect to } \eta)
$$

in these coordinates. To eliminate the higher order terms in (20) we will use the following lemma.

Lemma 3. Let us consider a domain $U$ with coordinates $\left(\xi^{a}, \eta^{a}, \zeta^{\alpha}\right)$ and an odd vector field $A$ in $U$ satisfying $\left\{Q_{0}, A\right\}=0$ and vanishing for $\eta^{a}=0$. Then $A$ can be represented as $A=\left[Q_{0}, B\right]$ where $B$ is an even vector field in $U$ such that $B=0$ for $\eta^{a}=0$.

Here we used the notation $Q_{0}$ for an odd vector field corresponding to the operator $\hat{Q}_{0}=\eta^{a} \partial / \partial \xi^{a}$. This vector field determines a $Q$-structure on $U$ and the lemma shows that an infinitesimal deformation of this $Q$-structure is trivial (i.e. it leads to an equivalent $Q$-structure) if the field $Q$ in the deformed $Q$ structure has the same zeros as $Q_{0}$. To prove the lemma we introduce notations

$$
\begin{aligned}
& \hat{A}=A^{a} \frac{\partial}{\partial \xi^{a}}+\tilde{A}^{a} \frac{\partial}{\partial \eta^{a}}+A^{\alpha} \frac{\partial}{\partial \zeta^{\alpha}}, \\
& \hat{B}=B^{a} \frac{\partial}{\partial \xi^{a}}+\tilde{B}^{a} \frac{\partial}{\partial \eta^{a}}+B^{\alpha} \frac{\partial}{\partial \zeta^{\alpha}}
\end{aligned}
$$

and note that the equations $\left\{Q_{0}, A\right\}=0, A=\left[Q_{0}, B\right]$ can be written as

$$
\begin{gathered}
\hat{Q}_{0} \tilde{A}^{a}=0, \hat{Q}_{0} A^{\alpha}=0, \hat{Q}_{0} A^{a}-\tilde{A}^{a}=0, \\
\tilde{A}^{a}=\hat{Q}_{0} \tilde{B}^{a}, A^{\alpha}=\hat{Q}_{0} B^{\alpha}, A^{a}=\hat{Q}_{0} B^{a}+\tilde{B}^{a} .
\end{gathered}
$$


We see that one can take $\tilde{B}^{a}=A^{a}, B^{a}=0$. To find $B^{\alpha}$ we take into account that $\hat{Q}_{0}$ can be consider as an exterior differential and use the Poincare lemma.

The lemma can be used to eliminate the higher order terms with respect to $\eta^{a}$ in (20) by means of an appropriate change of coordinates in a neighborhood of $Y$. One can give a heuristic proof of this statement eliminating the second order terms, then the third order terms and so on. To get a rigorous proof one can write an equation for the change of coordinates we are interested in and prove the existence of a solution to this equation using the implicit function theorem and Lemma 3. (This Lemma gives us the information about the solution to the equation obtained by means of linearization of the equation at hand.)

We proved Theorem 2. The same arguments can be applied to prove: Theorem $2^{\prime}$. Let us suppose that $X$ is a manifold provided with a $Q$-structure, $Y=\{x \in X \mid Q x=0\}$ and $H_{x}=H\left(T_{x}, Q_{x}\right)=0$ for $x \in Y$. Then in a neighborhood of $Y$ this $Q$-structure is equivalent to the standard $Q$-structure on $T Y$.

\section{4. $S$-manifolds and $Q S$-manifolds.}

To determine a volume element in a manifold $X$ we have to choose volume elements in all tangent spaces $T_{x} X, x \in X$. (We suppose that these volume elements depend smoothly on $x \in X$.) We will say that a volume element in the manifold $X$ specifies an $S$-structure on $X$. In local coordinates $z^{1}, \ldots, z^{n}$ a volume element is determined by an even invertible function $\rho(z)$ (density). By a change of local coordinates the density gains a factor equal to a (super)Jacobian.

The $S$-structure on $X$ determines an operator div acting from the space of vector fields in $X$ into the space of functions on $X$. In local coordinates

$$
\operatorname{div} A=\rho^{-1} \frac{\partial_{r}}{\partial z^{a}}\left(\rho A^{a}\right)=\frac{\partial_{r} A^{a}}{\partial z^{a}}+\frac{\partial_{r} \ln \rho}{\partial z^{a}} A^{a}
$$

If the manifold $X$ is provided with a $Q$-structure and an $S$-structure simultaneously and the $S$-structure is $Q$-invariant (i.e. $\operatorname{div} Q=0$ ) we will talk about a $Q S$-structure on $X$.

As we mentioned above, if $X$ is provided with $Q$-structure the subspaces $B_{x} \subset T_{x} Y$ determine a foliation of $Y=\{x \in X \mid Q x=0\}$. Let us suppose that this foliation is a fibration of $Y$; the base of this fibration will be denoted by $R$. The set $C(R)$ of all functions on $R$ can be identified with the set of functions $\varphi$ on $Y$ satisfying $\hat{b} \varphi=0$ for all vector fields satisfying $b(x) \in B_{x}, x \in Y$ (i.e. for $b \in \mathcal{B}$ ). The set $V$ ect $(R)$ of all vector fields on $R$ can be identified with the set of vector fields $A$ on $Y$ satisfying $[b, A\} \in \mathcal{B}$ for every $b \in \mathcal{B}$. If $x \in Y$ the natural projection $\pi$ of $Y$ onto $R$ induces a linear map $\pi_{*}^{x}$ of the tangent space $T_{x} Y=Z_{x}$ onto the tangent space $T_{\pi(x)} R$ and the kernel of $\pi_{*}^{x}$ coincides with $B_{x}$. This means that exists a natural one-to-one linear map from the homology group $H\left(T_{x}, Q_{x}\right)=Z_{x} / B_{x}$ onto the tangent space $T_{\pi(x)} R$. Recall that there exists a one-to-one correspondence Tor between volume elements 
in $T_{x}=T_{x}(X)$ and volume elements in $H\left(T_{x}, Q_{x}\right)$. If $X$ is a $Q S$-manifold this correspondence gives us a volume element in $T_{r} R$ for every $r \in R$ by means of the identification $T_{r} R=H\left(T_{x}, Q_{x}\right)$ where $\pi(x)=r$. We will prove that the volume element in $T_{r} R$ does not depend on the choice of $x$ and therefore that $R$ has a natural $S$-structure. It is sufficient to check that the identifications $T_{r} R=H\left(T_{x}, Q_{x}\right)$ and $T_{r} R=H\left(T_{\tilde{x}}, Q_{\tilde{x}}\right)$ generate the same volume element in $T_{r} R$ in the case when $\tilde{x}$ belongs to $\mathrm{w}$ a neighborhood $U$ of $x \in Y$ such that we can apply Theorem 1. The assumption $\operatorname{div} \hat{Q}=0$ leads to the equation $\hat{Q}_{0} \rho=0$ in the coordinates $(\xi, \eta, \zeta)$. (Here $\hat{Q}_{0}=\eta^{a} \partial / \partial \xi^{a}$.) Writing the density $\rho$ in the form $\rho=\rho_{0}(\xi, \zeta)+\tilde{\rho}(\xi, \eta, \zeta)$ where $\tilde{\rho}(\xi, \eta, \zeta)=0$ for $\eta=0$ we obtain from $\hat{Q}_{0} \rho=0$ that $\rho_{0}(\xi, \zeta)$ does not depend on $\xi$. Independence of the volume element in $T_{r} R$ on the choice of $x$ follows immediately from this fact.

Let us describe the set $C(R)$ of functions on $R$, the set $V e c t(R)$ of vector fields on $R$ and the operator div : $\operatorname{Vect}(R) \rightarrow C(R)$ corresponding to the natural $S$-structure on $R$ in terms of functions and vector fields on $X$. The operator $\hat{Q}$ can be considered as a differential acting on the space of functions on $X$ and therefore we can consider the homology group $\mathcal{H}^{0}(X)=\mathcal{Z}^{0}(X) / \mathcal{B}^{0}(X)$ where $\mathcal{Z}^{0}(X)$ consists of $Q$-closed functions (i.e. functions satisfying $\hat{Q} f=0$ ) and $\mathcal{B}^{0}(X)$ consists of $Q$-exact functions (functions of the form $\hat{Q} \varphi$ ). Analogously, the (super)commutator with $\hat{Q}$ can be considered as a differential in the space of vector fields on $X$ and we can consider corresponding homology group $\mathcal{H}^{1}(X)=$ $\mathcal{Z}^{1}(X) / \mathcal{B}^{1}(X)$. (Here $\mathcal{Z}^{1}(X)$ consists of vector fields $A$ satisfying $[Q, A\}=0$ and $\mathcal{B}^{1}(X)$ consists of vector fields of the form $\left.[Q, A\}\right)$. A function $f \in \mathcal{Z}^{0}(X)$ restricted to $Y$ can be considered as a function on $R$ because $\hat{b} f=0$ for every vector field $b \in \mathcal{B}$. (Locally one can represent $b \in \mathcal{B}$ as a restriction of $[Q, a\}$ and therefore $b f=\hat{Q} \hat{a} f$ vanishes on $Y$.) Functions belonging to $\mathcal{B}^{0}(X)$ vanish on $Y$ therefore the construction above determines a linear map from $\mathcal{H}^{0}(X)$ into $C(R)$; we will denote this map by $\rho_{0}$. Similarly the restriction of a vector field $A \in \mathcal{Z}^{1}(X)$ to $Y$ determines a vector field on $R$ and the restriction of a vector field $A \in \mathcal{B}^{1}(X)$ vanishes on $R$. Hence we have a linear map from $\mathcal{H}^{1}(X)$ into $V e c t(R)$; this map will be denoted by $\rho_{1}$. It is easy to check that for every point $x \in Y$ there exists a neighborhood $U$ of $x$ in $X$ such that the maps $\rho_{0}$ and $\rho_{1}$ determine isomorphisms $\mathcal{H}^{0}(U)=C\left(R_{U}\right)$ and $\mathcal{H}^{1}(U)=\operatorname{Vect}\left(R_{U}\right)$. (Note that the neighborhood $U$ always can be chosen in such a way that the foliation of $Y \cap U$ is a fibration; the base of this fibration is denoted by $R_{U}$.) This fact follows immediately from Theorem 1 and the Poincare lemma. For example to prove that $\rho_{1}$ is an isomorphism we conclude from (23), (24) that every vector field belonging to $\mathcal{Z}^{1}(U)$ is homologous to a vector field (21) with $A^{a}=0, \tilde{A}^{a}=0, Q_{0} A^{\alpha}=0$ and that such a vector field belongs to $\mathcal{B}^{1}(U)$ iff $A^{\alpha}$ can be represented in the form $A^{\alpha}=\hat{Q}_{0} B^{\alpha}$. This remark permits us to identify $\mathcal{H}^{1}(U)$ with the space of functions $A^{\alpha}$ depending only on $\zeta$ (i.e. with $V e c t(R)$ ).

It follows from $\operatorname{div} \hat{Q}=0$ that the operator div maps $\mathcal{Z}^{1}(X)$ into $\mathcal{Z}^{0}(X)$ and $\mathcal{B}^{1}(X)$ into $\mathcal{B}^{0}(X)$. (The first of these facts is geometrically obvious. Re- 
ally $\mathcal{Z}^{1}(X)$ consists of $Q$-invariant vector fields and the $S$-structure on $X$ is $Q$-invariant. Therefore div maps $\mathcal{Z}^{1}(X)$ into the space $\mathcal{Z}^{0}(U)$ of $Q$-invariant functions on $X$. Both facts can be checked by means of direct calculation.) We see that the operator div acting from $V e c t(X)$ into $C(X)$ induces an operator acting from $\mathcal{H}^{1}(X)$ into $\mathcal{H}^{0}(X)$; let us denote this operator by div $v_{\mathcal{H}}$.

Lemma 4. In the case $X=U$ the operator $d i v_{\mathcal{H}}$ coincides with the operator div : Vect $\left(R_{U}\right) \rightarrow C\left(R_{U}\right)$ corresponding to the $S$-structure induced in $R_{U}$. (We use the identifications $\left.\mathcal{H}^{1}(U)=\operatorname{Vect}\left(R_{U}\right), \mathcal{H}^{0}(U)=C\left(R_{U}\right)\right)$. In the general case we have a commutative diagram

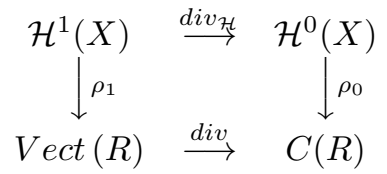

The first statement follows immediately from the consideration above. The second statement can be derived from the first one.

Lemma 5. If a function $f$ on a $Q S$-manifold $X$ can be represented in the form $f=Q \varphi$ (i. e. $f \in \mathcal{B}^{0}(X)$ ), then the integral of $f$ over $X$ vanishes. If $Y$ is empty the same is true for every $Q$-invariant function $f$ on $X$ (i. e. for every function $\left.f \in \mathcal{Z}^{0}(X)\right)$.

The second statement of the Lemma was proved in [5]. It follows immediately from the fact that in the case at hand we can find a coordinate system in $X$ such that $Q=\partial / \partial \xi^{a}$ where $\xi^{a}$ is one of the odd coordinates. One can conclude from this statement that in the case when $Y$ is not empty the integral of $f$ over $X$ depends only on values of $f$ and its derivatives at the points of $Y$; in other words this integral is determined by the function $f$ in an infinitesimal neighborhood of $Y$ (see[5]).

The first statement can be proved easily in the case when the function $\varphi$ vanishes outside a compact set $K \subset U$ where $U$ satisfies the conditions of Theorem 2 (i.e. $Q$ has the standard form (19) in $U$ ). One can reduce the general case to the simplest one using Theorem 2 and a partition of unity.

\section{5. $P$-manifolds and $S P$-manifolds.}

Let is consider a (super)manifold $M$ with an odd closed 2-form $\omega$. We will say that $M$ is a $P$-manifold (an odd symplectic manifold) if the form $\omega$ is nondegenerate. (The form $\omega=\frac{1}{2} d z^{i} \omega_{i j}(z) d z^{j}$ determines an odd inner product $\omega(a, b)=\frac{1}{2} a^{i} \omega_{i j}(z) b^{j}$ in the tangent space $T_{x} M$ at every point $x \in M$. The form $\omega$ is non-degenerate if this inner product is non-degenerate for all $x \in M$.) One can weaken the requirement of non-degeneracy of $\omega$ assuming that for every $x \in M$ the space $B_{x}=\left(T_{x} M\right)^{\perp}$ of vectors $b \in T_{x} M$ satisfying $\omega(a, b)=0$ for all $a \in T_{x} M$ is a superspace having dimension independent of $x$. In this case we will say that $M$ is a pre- $P$-manifold (an odd pre-symplectic manifold). One can prove that in a neighborhood of every point of a pre- $P$-manifold $M$ there exists 
a local coordinate system $\left(\xi^{a}, \eta_{a}, \zeta^{\alpha}\right)$ such that $\omega=d \xi^{a} d \eta_{a}$. (The coordinates $\left(\xi^{a}, \eta_{a}, \zeta^{\alpha}\right)$ are called Darboux coordinates.) It follows immediately from this fact that subspaces $B_{x} \subset T_{x} M$ determine a foliation of $M$; this foliation will be denoted by $\beta$. Let us say that a vector field $b$ on $M$ belongs to the set $\mathcal{B}$ if $b(x) \in B_{x}$ for every $x \in M$. A function $F$ on $M$ is a constant on every leaf of the foliation $\beta$ iff it is $\mathcal{B}$-invariant (i.e. $\hat{b} F=0$ for every $b \in \mathcal{B}$ ). If the foliation is a fibration one can identify the set of $\mathcal{B}$-invariant functions on $M$ with the set of functions on the base $M^{\prime}$ of this fibration. It is easy to check that the form $\omega$ induces a non-degenerate closed 2-form $\tilde{\omega}$ on $M^{\prime}$, therefore $M^{\prime}$ can be considered as a $P$-manifold; we say that this $P$-manifold corresponds to the pre- $P$-manifold $M$. (The form $\tilde{\omega}$ is determined by the condition $\omega=\pi^{*} \tilde{\omega}$ where $\pi$ denotes the natural projection of $M$ onto $M^{\prime}$.)

If the foliation $\beta$ is not a fibration we cannot construct a $P$-manifold $M^{\prime}$ corresponding to the pre- $P$-manifold $M$ but for every point $x \in M$ we can find a neighborhood $U$ such that a $P$-manifold $U^{\prime}$ does exist.

We say that $L$ is a Lagrangian submanifold of a pre- $P$-manifold $M$ if $L$ is a maximal isotropic submanifold of $M$ transversal to $B_{x}$ at every point $x \in L$. (We say that a submanifold $L$ is isotropic if for every two vectors $a, b \in T_{x} L$ we have $\omega(a, b)=0$. The transversality condition means that $T_{x} L \cap B_{x}=\emptyset$ for all $x \in L$.) For every $x \in M$ the space $T_{x} M / B_{x}$ is provided with a non-degenerate inner product; it is obvious that $L$ is a Lagrangian submanifold of $M$ iff the natural map of $T_{x} L$ into $T_{x} M / B_{x}$ is an isomorphism and its image is a maximal isotropic subspace. If the foliation $\beta$ is a fibration then the image $\pi(L)$ of $L$ under the natural projection $\pi: M \rightarrow M^{\prime}$ is a Lagrangian submanifold of $M^{\prime}$. (More precisely $\pi$ determines a Lagrangian immersion of $L$ into $M^{\prime}$ because $\pi(L)$ can have self-intersections.)

One can characterize Lagrangian submanifolds of a $(n \mid n)$-dimensional $P$ manifold $M$ as $(k, n-k)$-dimensional isotropic submanifolds of $M$.

It is easy to verify that $L$ is a Lagrangian submanifold of a pre- $P$-manifold $M$ iff for every point $x \in L$ one can find a basis $\left(e^{1}, \ldots, e^{n}, f_{1}, \ldots, f_{n}, g^{1}, \ldots g^{l}\right)$ of $T_{x} M$ such that $e^{1}, \ldots, e^{n}$ is a basis of $T_{x} L \subset T_{x} M$,

$$
\omega\left(e^{i}, e^{j}\right)=\omega\left(f_{i}, f_{j}\right)=\omega\left(e^{i}, g^{\alpha}\right)=\omega\left(f_{i}, g^{\alpha}\right)=\omega\left(g^{\alpha}, g^{\beta}\right)=0, \omega\left(e^{i}, f_{j}\right)=\delta_{j}^{i}
$$

(Here $1 \leq i, j \leq n, 1 \leq \alpha, \beta \leq l$.)

If $M$ is a pre- $P$-manifold then the inner product in $T_{x} M$ permits us to lower indices. In other words, if we have a vector field $A^{k}$ we can construct a covector field $A_{k}=\omega_{k l} A^{l}$. If $M$ is a $P$-manifold the correspondence between vector and covector fields is invertible: there exists an inverse matrix $\omega^{k l}$ for the matrix $\omega_{a b}$ and we assign a vector field $A^{k}=\omega^{k l} A_{l}$ to every covector field $A_{l}$. Hence for every function $H$ on $M$ we can construct a vector field $h^{a}=\omega^{a b} \partial_{l} H / \partial z^{b}$ (a Hamiltonian vector field with the Hamiltonian $H$ ). We get a map of the set $C(M)$ of functions on $M$ into the set $V e c t(M)$ of vector fields on $M$; this map will be denoted by $K$. Let us define the Poisson brackets $\{G, H\}$ of two 
functions on $M$ by the formula

$$
\{G, H\}=\frac{\partial_{r} G}{\partial z^{a}} \omega^{a b} \frac{\partial_{l} H}{\partial z^{b}} .
$$

Using the brackets (26) we can write the first order differential operator $\hat{h}$ corresponding to a vector field $h=K(H)$ as

$$
\hat{h} G=\{G, H\}=\frac{\partial_{r} G}{\partial z^{a}} h^{a}=h^{a} \frac{\partial_{l} G}{\partial z^{a}} .
$$

The set $C(M)$ can be considered as a Lie superalgebra with respect to the operation (26) and $K$ is a (parity reversing) homomorphism of this superalgebra into the Lie superalgebra $\operatorname{Vect}(M)$. More precisely, brackets (26) obey

$$
\begin{gathered}
\{G, H\}=-(-1)^{\left(\varepsilon_{G}+1\right)\left(\varepsilon_{H}+1\right)}\{H, G\} \\
(-1)^{\left(\varepsilon_{F}+1\right)\left(\varepsilon_{H}+1\right)}\{F,\{G, H\}\}+\text { cyclic perm. } F, G, H=0
\end{gathered}
$$

(graded anticommutativity and Jacobi identity). The operator $K$ transforms brackets into a graded (anti)commutator of vector fields.

The transformations preserving an odd closed 2-form $\omega$ are called $P(\omega)$ transformations or simply $P$-transformations. As we mentioned above a pre$P$-manifold $M$ can be covered with coordinate systems $\left(\xi_{(i)}^{a}, \eta_{a}^{(i)}, \zeta_{(i)}^{\alpha}\right)$ in such a way that the form $\omega$ specifying the pre- $P$-structure on $M$ has the standard form

$$
\omega_{0}=d \xi_{(i)}^{a} d \eta_{a}^{(i)}
$$

in these coordinates. Hence a pre- $P$-manifold can be pasted together from superdomains by means of $P\left(\omega_{0}\right)$-transformations. This fact can be considered an alternative definition of pre- $P$-manifold.

A one-to-one map $F$ of a domain $U$ with coordinates $\left(\xi^{a}, \eta_{a}, \zeta^{\alpha}\right)$ into a domain $\tilde{U}$ with coordinates $\left(\tilde{\xi}^{a}, \tilde{\eta}_{a}, \tilde{\zeta}^{\alpha}\right)$ will be called an $S P$-transformation if it is a $P$-transformation (i.e. $F^{*} \tilde{\omega}_{0}=\omega_{0}$, where $\omega_{0}=d \xi^{a} d \eta_{a}, \tilde{\omega}_{0}=d \tilde{\xi}^{a} d \tilde{\eta}_{a}$ ) and the determinant of the matrix

$$
\left(\begin{array}{cc}
\partial \tilde{\xi}^{a} / \partial \xi^{b}, & \partial \tilde{\eta}_{a} / \partial \xi^{b} \\
\partial \tilde{\xi}^{a} / \partial \eta_{b}, & \partial \tilde{\eta}_{a} / \partial \eta_{b}
\end{array}\right)
$$

is equal to 1 . It is easy to check that the $P$-transformation $F: U \rightarrow \tilde{U}$ induces a $P$-transformation $F^{\prime}: U^{\prime} \rightarrow \tilde{U}^{\prime}$ of corresponding $P$-manifolds; the unimodularity of the matrix (30) means that the Jacobian of $F^{\prime}$ is equal to 1 . We define a pre- $S P$-manifold $M$ as a manifold pasted together from domains $U_{i}$ with coordinates $\left(\xi_{(i)}^{a}, \eta_{a}^{(i)}, \zeta_{(i)}^{\alpha}\right)$ by means of $S P$-transformations. A pre- $S P$-manifold $M$ will be called an $S P$-manifold if $M$ is a $P$-manifold. In other words an $S P$ manifold can be pasted together from domains with coordinates $\left(\xi_{(i)}^{a}, \eta_{a}^{(i)}\right)$ by 
means of $S P$-transformations (i.e. $P$-transformations with Jacobian equal to 1 ). If there exists a $P$-manifold $M^{\prime}$ corresponding to the pre- $S P$-manifold $M$ then a pre- $S P$-structure on $M$ determines an $S P$-structure on $M^{\prime}$ and conversely an $S P$-structure on $M^{\prime}$ determines a pre- $S P$-structure on $M$. There exists a natural volume element (an $S$-structure) in an $S P$-manifold. If a manifold $M$ is provided with a $P$-structure and an $S$-structure simultaneously we introduce an operator $\Delta=\frac{1}{2} d i v \cdot K$ on the space $C(M)$ as a composition of the operators $K: C(M) \rightarrow$ Vect $M$ and div $:$ Vect $M \rightarrow C(M)$. For an $S P$-manifold we have $\Delta^{2}=0$. One can prove that conversely in the case when $\Delta^{2}=0$ a manifold provided with a $P$-structure and an $S$-structure is an $S P$-manifold [2].

If $M$ is a pre- $S P$-manifold we can define the operator $\Delta$ as an operator acting on the space $C(M, \mathcal{B})$ of $\mathcal{B}$-invariant functions on $M$. If there exists an $S P$-manifold $M^{\prime}$ corresponding to $M$ this fact follows from the identification $C(M, \mathcal{B})=C\left(M^{\prime}\right)$. In the general case we can use the fact that for every point $x \in M$ there exists a neighborhood such that $C(U, \mathcal{B})=C\left(U^{\prime}\right)$.

If $L$ is a Lagrangian submanifold of an pre- $S P$-manifold $M$ one can introduce a volume element (an $S$-structure) in $L$ as follows. If $e^{1}, \ldots, e^{n}$ is a basis in the tangent space $T_{x} L, x \in L$, we extend it to a basis $e^{1}, \ldots, e^{n}, f_{1}, \ldots, f_{n}, g^{1}, \ldots, g^{l}$ of $T_{x} M$ satisfying (25). Then the projections $\pi_{x} e^{1}, \ldots, \pi_{x} e^{n}, \pi_{x} f_{1}, \ldots, \pi_{x} f_{n}$ of the first $2 n$ vectors of this basis constitute a basis in $T_{\pi(x)} M^{\prime}$. (Recall that $\pi$ denotes the natural projection of $M$ onto the $S P$-manifold $M^{\prime}$ corresponding to the pre- $S P$-manifold $M$ ). We define the measure in $T_{x} L$ by the formula

$$
\lambda\left(e^{1}, \ldots, e^{n}\right)=\mu\left(\pi_{x} e^{1}, \ldots, \pi_{x} e^{n}, \pi_{x} f_{1}, \ldots, \pi_{x} f_{n}\right)^{1 / 2}
$$

where $\mu$ is the measure in $T_{\pi(x)} M^{\prime}$. (It is possible that the $S P$-manifold $M^{\prime}$ does not exist. In this case we have to modify the definition above by replacing $M$ with a neighborhood $U$ of $x \in L$ such that $U^{\prime}$ does exist.)

The formula (31) determines the volume element in $L$ up to a sign; the choice of the sign is discussed in [2].

One can prove the following statement.

Lemma 6. If $M$ is a pre-SP-manifold, $L$ is a Lagrangian submanifold, $H \in C(M, \mathcal{B})$ and $\Delta H=0$ then

$$
\int_{L_{1}} H d \lambda_{1}=\int_{L_{2}} H d \lambda_{2}
$$

where $L_{1}$ and $L_{2}$ are Lagrangian submanifolds of $M$ such that $m\left(L_{1}\right)$ is homologous to $m\left(L_{2}\right)$ in $m(M)$. If there exists an $S P$-manifold $M^{\prime}$ corresponding to $M$ the Lemma follows immediately from the identification $C(M, \mathcal{B})=C\left(M^{\prime}\right)$ and from the corresponding statement for $S P$-manifold (see[2]).

If the function $H$ is represented in the form $H=\exp \left(-\hbar^{-1} S\right)$ the equation $\Delta H=0$ is equivalent to the equation

$$
\frac{1}{2}\{S, S\}=\hbar \Delta S
$$


The equation (32) is called the quantum master equation and the equation

$$
\{S, S\}=0
$$

is known as the classical master equation or simply the master equation. If the function $H=\exp \left(-\hbar^{-1} S\right)$ obeys $\Delta H=0$ for all $\hbar$ then

$$
\{S, S\}=0, \Delta S=0
$$

\section{6. $Q S$-manifolds, $Q S P$-manifolds and the master equation.}

Let us suppose that a $P$-manifold $X$ is provided with an odd vector field $Q$ satisfying $\{Q, Q\}=0$ (with a $Q$-structure). We will say that $X$ is a $Q P$ manifold if the $P$-structure is specified by an odd non-degenerate $Q$-invariant 2 -form $\omega$; this means that the Lie derivarive $L_{Q} \omega$ of $\omega$ with respect to the vector field $Q$ vanishes: $L_{Q} \omega=0$. In terms of the odd Poisson bracket $\{F, G\}$ on $X$ the $Q$-invariance of the $P$-structure means that

$$
\hat{Q}\{F, G\}=\{Q F, G\}+\{F, \hat{Q} G\}(-1)^{\varepsilon_{F}+1}
$$

where $\hat{Q}$ stands for first order differential operator $Q^{a} \partial a$. Every solution $S$ to the master equation $\{S, S\}=0$ on $X$ determines a parity reversing first order differential operator $\hat{Q}=\hat{Q}_{S}$ by the formula

$$
\hat{Q} F=\{F, S\} .
$$

It satisfies $\hat{Q}^{2}=0$; therefore $S$ determines a $Q$-structure on $X$. It is easy to check that (34) for the operator (35) follows from the Jacobi identity (29). Hence every solution to the master equation determines a $Q P$-structure on $X$. Conversely, at least locally every first order differential operator $\hat{Q}$ satisfying (34) can be represented in the form (35). (The assertion that an operator $\hat{Q}$ obeying (34) can be represented in the form (35) with multivalued $S$ can be considered as a superanalog of the well known fact that a vector field preserving the Poisson brackets on a symplectic manifold can be obtained from a multivalued Hamiltonian.) We see that every $Q P$-structure on a $P$-manifold $X$ corresponds to a multivalued solution of the master equation.

To be more precise, if $\hat{Q}^{2}=0$ then for the corresponding $S$ we have $\{S, S\}=$ $\gamma$ where $\gamma$ is an odd constant. However $\gamma=0$ if $S$ has at least one critical point or if we don't have auxiliary odd parameters. (In mathematical language this means that $S$ is a superfunction on a supermanifold. Physicists speaking about a supermanifold often have in mind a family of supermanifolds; i. e. they allow dependence of all functions on auxiliary parameters.)

Let us suppose that a manifold $X$ is provided with an $S P$-structure and that $S$ is a solution of the master equation $\{S, S\}=0$ satisfying the condition 
$\Delta S=0$. One can prove that in this case the operator $\hat{Q}=\hat{Q}_{S}$ anticommutes with $\Delta$. The proof is based on the formula [3]

$$
\Delta(F \cdot G)=\Delta F \cdot G+(-1)^{\varepsilon(F)} F \cdot \Delta G+(-1)^{\varepsilon(F)}\{F, G\} .
$$

Taking $F=S$ and applying $\Delta$ to (36) we obtain

$$
0=\Delta(S \cdot \Delta G)+\Delta\{S, G\}
$$

Applying (36) once more we get

$$
0=\{S, \Delta G\}+\Delta\{S, G\}
$$

In other words,

$$
\hat{Q} \Delta+\Delta \hat{Q}=0
$$

Conversely, if an odd first order differential operator $\hat{Q}$ anticommutes with $\Delta$ then it corresponds to a function $S$ satisfying the master equation and the condition $\Delta S=0$. One can say that such an operator $Q$ preserves the $S P$ structure. Using this interpretation we can give a little bit different proof of (38). Namely the condition $\Delta S=0$ can be written in the form $\operatorname{div} Q=0$ and therefore can be interpreted as $Q$-invariance of the $S$-structure on $X$. Taking into account that $Q$-invariance of the $P$-structure on $X$ follows from the master equation we see that $\Delta$ is $Q$-invariant i.e. $\{Q, \Delta\}=0$.

We will use the term $Q S P$-manifold for an $S P$-manifold with a $Q$-structure if the operators $\hat{Q}$ and $\Delta$ anticommute.

Let us consider now the set $Y$ of all critical points of a function $S$ satisfying the master equation $\{S, S\}=0$ on a $P$-manifold $X$. In other words we consider a $Q P$-manifold $X$ and the set $Y=\{x \in X \mid Q x=0\}$. As in every $Q$-manifold for every point $x \in Y$ we have an odd linear operator $Q_{x}$ in $T_{x} X$ satisfying $Q_{x}^{2}=0$. The $P$-structure on $X$ determines an inner product $\omega(a, b)=\frac{1}{2} a^{i} \omega_{i j} b^{j}$ in $T_{x} X$; it is easy to check that the operator $Q_{x}$ is skew-symmetric with respect to this inner product. Therefore the image $B_{x}$ of $Q_{x}$ is an orthogonal complement to the kernel $Z_{x}$ of $Q_{x}$ in this inner product. This means that the restriction of the inner product $\omega(a, b)$ to $Z_{x}$ is degenerate, but it induces a non-degenerate inner product in $H\left(T_{x}, Q_{x}\right)=Z_{x} / B_{x}$. We always consider the case when $Y$ is a manifold, $Z_{x}$ and $B_{x}$ are superspaces with dimension independent on $x \in Y$. In this case the restriction of the form $\omega$ to $Y$ determines the structure of an odd pre-symplectic manifold (a pre- $P$-structure) in $Y$. It follows from the facts mentioned above that the foliation of $Y$ generated by the $Q$-structure on $X$ coincides with the foliation induced by the pre- $P$-structure on $Y$. If the foliation of $Y$ specified by the subspaces $B_{x} \subset Z_{x}=T_{x}(Y)$ is a fibration we get a $P$-structure on the base $Y^{\prime}$ of this fibration.

Let us consider now an $S P$-manifold $X$ and a function $S$ on $X$ satisfying $\{S, S\}=0, \Delta S=0$. (Recall that these data determine a $Q S P$-structure on 
$X$.) In this case the manifold $Y^{\prime}$ is provided simultaneously with a $P$-structure because $X$ is a $Q P$-manifold and with a $S$-structure because $X$ is a $Q S$-manifold.

Theorem 3. The manifold $Y^{\prime}$ corresponding to a $Q S P$-manifold $X$ is an $S P$-manifold (i.e. the $S$-structure and $P$-structure on $Y^{\prime}$ determine an $S P$ structure on $Y^{\prime}$.)

To prove this theorem it is necessary to check that the operator $\Delta=\frac{1}{2} \mathrm{div} \cdot \mathrm{K}$ where div : $\operatorname{Vect}\left(Y^{\prime}\right) \rightarrow C\left(Y^{\prime}\right)$ is determined by the $S$-structure on $Y^{\prime}$ and $K: C\left(Y^{\prime}\right) \rightarrow \operatorname{Vect}\left(Y^{\prime}\right)$ is determined by the $P$-structure on $Y^{\prime}$ obeys $\Delta^{2}=0$. It is sufficient to check this fact locally. This means that we can replace $X$ by $U$ where $U$ is chosen in such a way that the maps $\rho_{0}$ and $\rho_{1}$ determine isomorphisms $\mathcal{H}^{0}(U)=C\left(U^{\prime}\right), \mathcal{H}^{1}(U)=V e c t\left(U^{\prime}\right)$.

It is easy to check that the map $K: C(U) \rightarrow V e c t(U)$ determined by the $P$ structure on $U$ transforms $\mathcal{Z}^{0}(U)$ into $\mathcal{Z}^{1}(U), \mathcal{B}^{0}(U)$ into $\mathcal{B}^{1}(U)$ and therefore generates a map $K_{\mathcal{H}}$ from $\mathcal{H}^{0}(U)$ into $\mathcal{H}^{1}(U)$. One can verify that after the identifications $\mathcal{H}_{0}(U)=C\left(U^{\prime}\right), \mathcal{H}^{1}(U)=V e c t\left(U^{\prime}\right)$ the map $K_{\mathcal{H}}$ coincides with the map $K: C\left(U^{\prime}\right) \rightarrow \operatorname{Vect}\left(U^{\prime}\right)$ determined by the $P$-structure on $U^{\prime}$. Now one can apply Lemma 4 to prove that the operator $\Delta_{\mathcal{H}}=\frac{1}{2} \operatorname{div}_{\mathcal{H}} \cdot K_{\mathcal{H}}$ coincides with $\Delta=\frac{1}{2}$ div $\cdot K$ after the identification $\mathcal{H}^{0}(U)=C\left(U^{\prime}\right)$. It is evident that $\Delta_{\mathcal{H}}^{2}=0$, hence $\Delta^{2}=0$ and $Y^{\prime}$ is an $S P$-manifold.

The same arguments lead to a little bit more general result valid also in the case when the foliation of $Y$ is not a fibration.

Theorem $3^{\prime}$. The manifold $Y$ corresponding to a $Q S P$-manifold $X$ is a pre- $S P$-manifold.

\section{Torsion in linear $P$-manifolds.}

Let suppose now that $E$ is provided with a non-degenerate odd differential form $\omega=\frac{1}{2} d z^{i} \omega_{i j} d z^{j}$ where $\omega_{i j}$ are constants. The form $\omega$ is obviously closed, so we can say that $E$ is provided with a $P$-structure. One can say that $E$ is a linear $P$-manifold (This means that the $P$-structure on $E$ is translationally invariant). A Lagrangian subspace $L$ of $E$ is by definition a maximal isotropic subspace of $E$ (i.e. a maximal subspace where $\omega$ vanishes: $\omega(z, \tilde{z})=0$ for $z \in L, \tilde{z} \in L)$. One can always find Darboux coordinates, i.e. a coordinate system $\left(x^{1}, \ldots, x^{n}, \xi_{1}, \ldots, \xi_{n}\right)$ in $E$ such that $\omega$ has the form

$$
\omega=d x^{i} d \xi_{i}
$$

where $x^{i}$ and $\xi_{i}$ have opposite parity; moreover for every Lagrangian subspace $L$ one can find Darboux coordinates in such a way that $L$ is singled out by the equations $\xi_{1}=\ldots=\xi_{n}=0$. The dimension of Lagrangian subspace is equal to $(k, n-k)$ where $0 \leq k \leq n$. If the notations are chosen in such a way that $x^{1}, \ldots, x^{n}$ are even, $\xi_{1}, \ldots \xi_{n}$ are odd, the Lagrangian subspace of dimension $(k, n-k)$ can be singled out in an appropriate coordinate system by equations $x^{k+1}=\ldots=x^{n}=0, \xi_{1}=\ldots=\xi_{k}=0$. The difference between the even dimension and the odd dimension of a Lagrangian subspace $L$ will be denoted 
by $d$ (i.e. $d=k-(n-k)=2 k-n)$.

One can apply the general theory of $P$-manifolds to the linear $P$-manifold $E$. In particular, one can define Poisson brackets and the Hamiltonian vector field $K_{S}$ corresponding to a function $S$.

If $S$ is an even quadratic function, the corresponding vector field $S=$ $\frac{1}{2} z^{i} S_{i j} z^{j}$ has the form

$$
K^{i}(z)=\omega^{i j} S_{j k} z^{k}=\hat{S}_{k}^{i} z^{k}
$$

where

$$
\hat{S}_{k}^{i}=\omega^{i j} S_{j k}
$$

can be considered as the matrix of the parity reversing linear operator $\hat{S}$ acting on $E$. In more invariant terms we can say that

$$
S(z)=\omega(z, \hat{S} z)
$$

The bilinear form $S(z, w)$ corresponding to the quadratic form $S(z)$ can be represented as

$$
S(z, w)=\frac{1}{2} z^{i} S_{i j} w^{j}=\omega(z, \hat{S} w)
$$

If $\{S, S\}=0$ it follows from (29) that $\hat{S}^{2}=0$ and therefore we can consider the homology group $H$ and the torsion. (We always suppose that $Z=\operatorname{Ker} \hat{S}$ and $B=\operatorname{Im} \hat{S}$ can be considered as linear (super) subspaces of $E$ ). One can say a quadratic function $S$ satisfying $\{S, S\}=0$ in a linear $P$-space $E$ determines a linear $Q P$-structure in $E$.

Let us fix a volume element $\mu$ in $E$. If $L$ is a Lagrangian subspace in $E$ then one can define a volume element in $L$ by the formula

$$
\lambda\left(e_{1}, \ldots, e_{n}\right)=\mu\left(e_{1}, \ldots, e_{n}, f^{1}, \ldots, f^{n}\right)^{1 / 2}
$$

where $e_{1}, \ldots, e_{n}$ constitute a basis in $L$ and $f^{1}, \ldots, f^{n}$ are vectors in $E$ satisfying $\omega\left(e_{i}, f^{j}\right)=\delta_{i}^{j}$. In the case when $L$ is singled out by equations $\xi_{1}=\ldots=\xi_{n}=0$ in Darboux coordinates and $\hat{e}_{i}=e_{i}^{j} \partial / \partial x^{j}$ we can take $f^{j}=e_{i}^{j} \partial / \partial \xi_{i}$. The proof of existence of vectors $f^{1}, \ldots, f^{n}$ for a general Lagrangian subspace can be reduced to this simplest case. Moreover the same arguments show that one can impose an additional requirement $\left(f^{i}, f^{j}\right)=0$ on vectors $f^{i}$; then these vectors will be determined uniquely. Note that (44) determines $\lambda$ only up to a sign and that (44) must be modified by a constant factor $\pm i$ in the case when the number part $m\left(\mu\left(e_{1}, \ldots, e_{n}, f^{1}, \ldots, f^{n}\right)\right)$ of $\mu\left(e_{1}, \ldots, e_{n}, f^{1}, \ldots, f^{n}\right)$ is negative. The ambiguity of sign will be neglected in all formulas below; therefore we omit also other irrelevant sign factors.

Lemma 7. Let $S$ denote a quadratic form of $E$ satisfying $\{S, S\}=0$ and $L$ denote a Lagrangian subspace of $E$ such that the restriction of $S$ to $L$ is a 
non-degenerate quadratic form on $L$. Then the homology group constructed by means of the operator $\hat{S}$ defined by (42) is trivial and

$$
\operatorname{Tor}^{\frac{1}{2}} \hat{S}=(2 \pi)^{d / 2} \int_{L} e^{-S} d \lambda
$$

where $\lambda$ denotes the volume element on $L$ corresponding to a volume element $\mu$ in $E$ and $\operatorname{Tor} \hat{S}$ denotes the image of $\mu$ by the isomorphism $\Lambda(E)=\Lambda(H)=$ $R^{1,0}$. (Recall that $d$ is the difference between the even dimension and the odd dimension of $L$.)

To prove the Lemma let us take a basis $e_{1}, \ldots, e_{n}$ satisfying

$$
\lambda\left(e_{1}, \ldots, e_{n}\right)=\mu\left(e_{1}, \ldots, e_{n}, f^{1}, \ldots, f^{n}\right)=1
$$

where $f^{i}$ obey $\omega\left(e_{i}, f^{j}\right)=\delta_{i}^{j}$ as above. The coefficients $a_{i j}$ in the decomposition

$$
\hat{S} e_{k}=f^{j} a_{j k}+e_{j} b_{k}^{j}
$$

can be calculated by means of (43). Namely

$$
a_{j k}=\omega\left(e_{j}, \hat{S} e_{k}\right)=S\left(e_{j}, e_{k}\right)
$$

We assume that the form $S$ is non-degenerate on $L$; therefore the matrix $a_{j k}$ is non-degenerate. We have some freedom in the choice of $f^{1}, \ldots, f^{n}$; namely we can replace $f^{i}$ by $f^{i}+k_{j}^{i} e_{j}$ and the condition $\omega\left(e_{j}, f^{i}\right)=\delta_{j}^{i}$ will not be violated. Utilizing the non-degeneracy of $a_{j k}$ one can check that this freedom is sufficient to get $b_{i}^{j}=0$. In such a way we obtain a basis $e_{1}, \ldots, e_{n}, f^{1}, \ldots, f^{n}$ on $E$ obeying $\hat{S} e_{k}=f^{j} a_{j k}$. It follows immediately from $\hat{S}^{2}=0$ and the non-degeneracy of $a_{j k}$ that $\hat{S} f^{j}=0$ for this basis. We obtain that the space $Z$ is spanned by $f^{1}, \ldots, f^{n}$ and coincides with $B$, and therefore $H=0$. One can apply (12) to calculate the torsion; we get

$$
\operatorname{Tor} \hat{S}=\mu\left(e_{1}, \ldots, e_{n}, \hat{S} e_{1}, \ldots, \hat{S} e_{n}\right)=(\operatorname{det} a)^{-1} \mu\left(e_{1}, \ldots, e_{n}, f^{1}, \ldots, f^{n}\right)=(\operatorname{det} a)^{-1}
$$

From the other side

$$
\int_{L} e^{-S} d \lambda=(\operatorname{det} S)^{-\frac{1}{2}}(2 \pi)^{-\frac{d}{2}}
$$

where $S_{i j}=S\left(e_{i}, e_{j}\right)$; we obtain (45) using (48).

One can generalize Lemma 1 to the case when the restriction $\tilde{S}$ of $S$ is degenerate on $L$. Then the space $N$ of critical points of $\tilde{S}$ is invariant with respect to $\hat{S}$ (this follows immediately from $\hat{S}^{2}=0$ ); we denote the operator $\hat{S}$ restricted to $N$ by $Q$. Let us consider the partition function $Z_{\tilde{S}, Q}$ of $\tilde{S}$ with respect to $Q$. In the case when the homology group of $Q$ in $N$ is trivial one can prove that the homology group of $\hat{S}$ in $E$ is trivial too and we can consider $Z_{\tilde{S}, Q}$ and the torsion Tor $\hat{S}$ as numbers. The generalization of (45) looks as follows:

$$
Z_{\tilde{S}, Q}=(2 \pi)^{d / 2} \operatorname{Tor}^{1 / 2} \hat{S}
$$


The same formula remains correct in some sense in the case when $Q$ has nontrivial homology in $N$. More precisely, one can prove

Lemma $7^{\prime}$. Let $S$ denote a quadratic form on $E$ satisfying $\{S, S\}=0$. If $N$ stands for the space of critical points of the restriction $\tilde{S}$ of $S$ to a Lagrangian subspace $L$ and $Q$ denotes the operator $\hat{S}$ restricted to $N$ then there exists a canonical isomorphism

$$
\Lambda(H(E, \hat{S}))=\Lambda(H(N, Q) \otimes H(N, Q))=\Lambda(H(N, Q))^{2}
$$

(Here $H(E, \hat{S})$ stands for the homology group of the operator $\hat{S}$ acting in $E$ and $H(N, Q)$ for the homology group of $Q$ acting on $N$.) Using the identification of $\Lambda\left(H(E, \hat{S})\right.$ and $\Lambda(H(N, Q))^{2}$ by means of (52) one can prove that

$$
(2 \pi)^{-d} \operatorname{Tor} \hat{S}=Z_{\tilde{S}, Q} \otimes Z_{\tilde{S}, Q}
$$

where $Z_{\tilde{S}, Q}$ denotes the partition function of $\tilde{S}$ with respect to $Q$ considered as an element of $\Lambda(H(N, Q))$.

To prove (53) we fix the basis $e_{1}, \ldots, e_{n}$ of $L$ in such a way that the first vectors $e_{1}, \ldots, e_{k}$ of this basis constitute a basis in $N$. The basis $e_{1}, \ldots, e_{n}$ of $L$ can be extended to a basis $e_{1}, \ldots, e_{n}, f^{1}, \ldots, f^{n}$ of $E$ by means of vectors $f^{1}, \ldots, f^{n}$ satisfying $\omega\left(f^{i}, f^{j}\right)=0, \omega\left(e_{j}, f^{i}\right)=\delta_{j}^{i}$.

The operator $\hat{S}$ corresponding to the quadratic functional $S$ has the form

$$
\begin{aligned}
& \hat{S} e_{i}=f^{j} a_{j i}+e_{j} b_{i}^{j} \\
& \hat{S} f^{i}=f^{j} c_{j}^{i}+e_{j} d^{j i}
\end{aligned}
$$

It follows from our assumptions that $a_{i j}=a_{j i}=0$ for $1 \leq i \leq k, 1 \leq j \leq$ $n$. The subspace $N$ spanned by $e_{1}, \ldots, e_{k}$ and the subspace $M$ spanned by vectors $e_{k+1}, \ldots, e_{n}, \hat{S} e_{k+1}, \ldots, \hat{S} e_{n}$ are invariant with respect to the operator $\hat{S}$. Therefore we can consider the coset space $F=E /(N+M)$ and the operator $\bar{S}$ induced by $\hat{S}$ in $F$. The vectors $\bar{f}^{1}, \ldots, \bar{f}^{k}$ obtained from $f^{1}, \ldots, f^{k}$ by means of natural projection $E \rightarrow F$ form a basis in $F$; it is easy to check that

$$
\bar{S} \bar{f}^{i}=\bar{f}^{j} c_{j}^{i} .
$$

It is easy to check that the matrix $c$ can be obtained from the matrix $b$ by means of (super)transposition and parity reversion. In invariant terms one can say that $F$ can be identified with $\Pi N^{*}$ and $\bar{S}=\Pi Q^{*}$ (recall that the operator $\hat{S}$ acting on $N$ is denoted by $Q$ ). We see that

$$
H(F, \bar{S})=\Pi H(N, Q)^{*}
$$

and therefore

$$
\Lambda H(F, \bar{S})=\Lambda H(N, Q)
$$


It is evident that the subspace $M$ is acyclic with respect to $\hat{S}$ (this follows from the non-degeneracy of $S$ on the subspace spanned by $\left.e_{k+1}, \ldots, e_{n}\right)$ and therefore $H(M+N, \hat{S})=H(N, Q)$. Now we can apply the exact homology sequence of the pair $(E, M+N)$ to obtain that in the case $H(N, Q)=0$ we have $H(E, \hat{S})=0$ and in the general case

$$
\Lambda H(E, \hat{S})=\Lambda H(M+N, \hat{S}) \otimes \Lambda H(F, \bar{S})=\Lambda H(N, Q) \otimes \Lambda H(N, Q) .
$$

To prove (53) we have to apply (15). We obtain

$$
\operatorname{Tor}(E, \hat{S})=\operatorname{Tor}(M+N, \hat{S}) \otimes \operatorname{Tor}(F, \bar{S})
$$

where the torsions are calculated with respect to volume elements determined by the bases in $E, M+N$ and $F$ that we have chosen. It is easy to check that

$$
\begin{gathered}
\operatorname{Tor}(M+N, \hat{S})=\operatorname{Tor}(M, \hat{S}) \cdot \operatorname{Tor}(N, Q), \\
\operatorname{Tor}(F, \bar{S})=\operatorname{Tor}(N, Q) \\
\operatorname{Tor}(M, \hat{S})=(\operatorname{det} \sigma)^{-1}
\end{gathered}
$$

where $\sigma$ denotes the matrix of the form $S$ on $M$ in the basis $e_{k+1}, \ldots, e_{n}$ (in other words $\left.\sigma_{i j}=\omega\left(e_{i}, S e_{j}\right), k+1 \leq i, j \leq n\right)$. Combining these equations with 60 ) we get (53).

Let us apply Lemma $7^{\prime}$ to the important particular case when the space $N$ of critical points of $\tilde{S}$ consists of critical points of $S$ (i. e. $N \subset Z=\operatorname{Ker} \hat{S}$ ). It is easy to check that in this case $Q=0$ and therefore $H(N, Q)=N$. We obtain

Lemma $7^{\prime \prime}$. Let us suppose that the quadratic form $S$ on $E$ satisfies $\{S, S\}=0$ and all critical points of the restriction $\tilde{S}$ to a Lagrangian subspace $L \subset E$ are critical points of $S$ on $E$. Then

$$
\Lambda(H(E, \hat{S}))=\Lambda(N) \otimes \Lambda(N)
$$

and

$$
(2 \pi)^{-d} \operatorname{Tor} \hat{S}=Z_{\tilde{S}} \otimes Z_{\tilde{S}}
$$

Here $Z_{\tilde{S}}$ denotes the partition function of $\tilde{S}$ considered as an element of $\Lambda(N)$. If $x^{1}, \ldots, x^{n}, \xi_{1}, \ldots, \xi_{n}$ are Darboux coordinates in $E$, then the function

$$
S=\xi_{i} \sigma^{i j} \xi_{j}
$$

obviously obeys $\{S, S\}=0$. One can prove that this form of $S$ is in some sense general.

Lemma 8. If $S$ is a quadratic function on $E$ satisfying $\{S, S\}=0$ then one can introduce Darboux coordinates $x^{1}, \ldots, x^{n}, \xi_{1}, \ldots, \xi_{n}$ in $E$ in such a way that $S$ depends only on $\xi_{1}, \ldots, \xi_{n}$.

To give the proof we restrict to $Z=\operatorname{Ker} \hat{S}$ the form $\omega$ specifying the $P$ structure on $E$. We get a degenerate odd form $\tilde{\omega}$ on $Z$; the space of null-vectors 
of $\tilde{\omega}$ coincides with $B=\operatorname{Im} \hat{S}$. Hence we can define a non-degenerate odd 2-form $\omega^{\prime}$ on $H=Z / B$; the form $\omega^{\prime}$ determines a linear $P$-structure on $H$. (Compare with the consideration of the more general case of an arbitrary $Q P$-manifold in Section 6.) Let us fix a Lagrangian linear subspace $\Lambda$ in $H$. It is easy to check that $L=\pi^{-1}(\Lambda)$ where $\pi$ stands for the natural projection of $Z$ onto $H$ is a Lagrangian subspace of $E$. Really, it is evident that $L$ is isotropic. Using the relations $l_{1}=\lambda_{1}+b_{1}, l_{2}=\lambda_{2}+b_{2}, h=z_{1}-b_{1}=z_{2}-b_{2}, n=z_{1}+b_{2}=$ $z_{2}+b_{1}$ we obtain $l_{1}+l_{2}=n$; hence $L$ is a Lagrangian subspace. (Here we used the notations $\operatorname{dim} E=(n, n), \operatorname{dim} Z=\left(z_{1}, z_{2}\right), \operatorname{dim} B=\left(b_{1}, b_{2}\right), \operatorname{dim} H=$ $\left.(h, h), \operatorname{dim} \Lambda=\left(\lambda_{1}, \lambda_{2}\right), \operatorname{dim} L=\left(l_{1}, l_{2}\right)\right)$. Now we can introduce Darboux coordinates $x^{1}, \ldots, x^{n}, \xi_{1}, \ldots, \xi_{n}$ in $E$ in such a way that $L$ is singled out by equations $\xi_{1}=\ldots=\xi_{n}=0$. It is easy to verify that $S$ depends only on $\xi_{1}, \ldots, \xi_{n}$ in this coordinate system.

One can reformulate Lemma 8 in a coordinate-free way.

Lemma $8^{\prime}$. If $S$ is a quadratic function on $E$ satisfying $\{S, S\}=0$ then one can find a Lagrangian subspace $M \subset E$ and a linear projection of $E$ onto $M$ in such a way that $S(x)=S(\mu(x))$ and the kernel of $\mu$ is a Lagrangian subspace of $E$.

To deduce this statement from Lemma 8 it is sufficient to single out $M$ by equations $x^{1}=\ldots=x^{n}=0$ and define $\mu$ by the formula

$$
\mu\left(x^{1}, \ldots, x^{n}, \xi_{1}, \ldots, \xi_{n}\right)=\left(0, \ldots, 0, \xi_{1}, \ldots, \xi_{n}\right) .
$$

It follows from our proof of Lemma 8 that $\operatorname{dim} M=(m, n-m)$ where $m$ is an arbitrary number obeying $b_{2} \leq m \leq n-b_{1}$.

Let us denote the set of critical points of the restriction of $S$ to $M$ by $\mathcal{K}$. It is easy to check that the set $Z$ of critical points of $S$ on $E$ can be represented in the form $Z=\rho^{-1}(\mathcal{K})$, in other words a point $e \in E$ satisfies $\hat{S} e=0$ if and only if $\mu(e) \in \mathcal{K}$.

Let us suppose that $\tilde{M}$ is a Lagrangian subspace of $E$ such that the projection $\mu$ considered as a map from $\tilde{M}$ into $M$ is an isomorphism. Then in the coordinate system $(x, \xi)$ where $\mu$ is given by (68) we can represent $\tilde{M}$ as a set of points $x^{1}, \ldots, x^{n}, \xi_{1}, \ldots, \xi_{n}$ where $\xi_{i}=\sigma_{i j} x^{j}, \sigma_{i j}$ is a fixed matrix. It follows immediately from the description of the set $Z$ that the critical points of $S$ considered as a function of $\tilde{M}$ belong to $Y$. This observation permits us to prove:

Lemma 9. The critical points of $S$ restricted to generic Lagrangian subspace of dimension $(m, n-m)$ where $b_{2} \leq m \leq n-b_{1}$ belong to the set $Z=\operatorname{Ker} \hat{S}$.

To give a proof of this Lemma we construct a Lagrangian subspace $M$ of dimension $(m, n-m)$ and a map $\mu: E \rightarrow M$ by means of Lemma $8^{\prime}$. Then it remains to note that for a generic Lagrangian subspace of the same dimension the projection $\mu$ of this subspace into $M$ is an isomorphism.

I am indebted to A. Givental, M. Kontsevich and E. Witten for useful discussions.

\section{References}


1. Batalin, I., Vilkovisky, G.: Gauge algebra and quantization. Physics Letters, 102B, 27(1981); Quantization of gauge theories with linearly dependent generators. Phys. Rev. D29, 2567(1983)

2. Schwarz, A.: Geometry of Batalin-Vilkovisky quantization. Commun. Math. Phys. (in press)

3. Witten, E.: A note on the antibracket formalism. Preprint IASSHS-HEP9019

4. Schwarz, A.: The partition function of a degenerate functional. Commun. Math. Phys. 67,1 (1979)

5. Witten, E.: The $N$ matrix model and gauged WZW models. Preprint IASSNS-HEP-91126

6.Dold, A.: Lectures on algebraic topology,1972,Springer 